BMJ Nutrition,

Prevention \& Health

\section{Overweight and obesity as risk factors for COVID-19-associated hospitalisations and death: systematic review and meta-analysis}

\author{
Wendemi Sawadogo (D) , Medhin Tsegaye, Andinet Gizaw, Tilahun Adera
}

To cite: Sawadogo W, Tsegaye M, Gizaw A, et al. Overweight and obesity as risk factors for COVID-19associated hospitalisations and death: systematic review and meta-analysis. BMJ Nutrition, Prevention \& Health 2022;0:e00375. doi:10.1136/ bmjnph-2021-000375

- Additional supplemental material is published online only. To view, please visit the journal online (http://dx.doi.org/10. 1136/bmjnph-2021-000375).

Division of Epidemiology, Department of Family Medicine and Population Health, Virginia Commonwealth University, Richmond, Virginia, USA

Correspondence to

Dr Wendemi Sawadogo, Division of Epidemiology, Department of Family Medicine and Population Health, Virginia Commonwealth University, Richmond, VA 232980101, USA;

sawadogow@vcu.edu

Received 23 September 2021 Accepted 22 December 2021

Check for updates

(C) Author(s) (or their employer(s)) 2022. Re-use permitted under CC BY-NC. No commercial re-use. See rights and permissions. Published by BMJ.

\section{ABSTRACT}

Objective To quantify the current weight of evidence of the association between overweight and obesity as risk factors for COVID-19-related hospitalisations (including hospital admission, intensive care unit admission, invasive mechanical ventilation) and death, and to assess the magnitude of the association and the potential doseresponse relationships.

Design PubMed, Embase, Cochrane, Web of Sciences, WHO COVID-19 database and Google Scholar were used to identify articles published up to 20 July 2021. Peerreviewed studies reporting adjusted estimates of the association between overweight or obesity and COVID-19 outcomes were included. Three authors reviewed the articles and agreed. The quality of eligible studies was assessed using the Newcastle-Ottawa Quality Assessment Scale. Random-effects meta-analysis was used to estimate the combined effects.

Results A total of 208 studies with 3550997 participants from over 32 countries were included in this metaanalysis. Being overweight was associated with an increased risk of COVID-19-related hospitalisations (OR $1.19,95 \% \mathrm{Cl} 1.12$ to $1.28, \mathrm{n}=21$ studies), but not death (OR 1.02, 95\% Cl 0.92 to $1.13, n=21$ ). However, patients with obesity were at increased risk of both COVID-19related hospitalisations (OR 1.72, 95\% $\mathrm{Cl} 1.62$ to 1.84 , $\mathrm{n}=58$ ) and death (OR 1.25, 95\% Cl 1.19 to $1.32, \mathrm{n}=77$ ). Similarly, patients with extreme obesity were at increased risk of COVID-19-related hospitalisations (OR 2.53, 95\% $\mathrm{Cl} 1.67$ to $3.84, \mathrm{n}=12)$ and death (OR $2.06,95 \% \mathrm{Cl}$ 1.76 to $3.00, n=19)$. There was a linear dose-response relationship between these obesity categories and COVID-19 outcomes, but the strength of the association has decreased over time.

Conclusion Being overweight increases the risk of COVID19-related hospitalisations but not death, while obesity and extreme obesity increase the risk of both COVID-19related hospitalisations and death. These findings suggest that prompt access to COVID-19 care, prioritisation for COVID-19 vaccination and other preventive measures are warranted for this vulnerable group.

\section{INTRODUCTION}

Recently, a novel coronavirus named SARSCoV-2, causing COVID-19, emerged in late 2019, and has posed a global threat with

\section{What this paper adds}

- The study found a linear dose-response relationship between obesity categories and COVID-19 outcomes, in which the risk of COVID-19-related outcomes steadily increased with increasing levels of obesity.

- Studies with the most recent data showed a weaker association between obesity and COVID-19 outcomes compared with the earlier days of the pandemic.

- This decrease in the strength of the association follows the pattern of the first wave of COVID-19: Asian-Pacific, Europe and North America.

devastating consequences. ${ }^{1}{ }^{2}$ To date, the disease is still spreading, new variants are emerging, breakthrough infections are being observed and the healthcare system is overwhelmed. $^{2}$ As of 14 September 2021, there were 226418035 infected cases resulting in 4 657736 deaths worldwide. ${ }^{3}$

The prognosis of COVID-19 has been consistently reported to worsen with older age, male sex and comorbidities such as diabetes, hypertension, chronic kidney disease, chronic respiratory disease and cardiovascular disease. ${ }^{4}$ These comorbidities are related to obesity and dysfunctional adipose tissue, thus predisposing people living with obesity to a greater risk of severe COVID-19. Studies have reported that adipose tissues are rich in ACE2 receptors, which act as a port of entry for SARS-CoV-2 to human cells. ${ }^{5}$ Additionally, obesity has been shown to alter immune function and increase the susceptibility to infection from different pathogens. ${ }^{6}$

More recently, several meta-analyses showed that obesity was associated with poor prognosis of COVID-19. ${ }^{78}$ However, these meta-analyses were limited by the number of included studies, ranging from 3 to 60, and mainly used body mass index (BMI) as a measure 
of obesity. Additionally, since the virus is changing, and the literature has been constantly evolving, it is important to reassess the risk of poor outcomes associated with obesity and summarise the current weight of evidence. This review aims to quantify the association between overweight or obesity and COVID-19-related hospitalisations (including hospital admission, intensive care unit (ICU) admission, invasive mechanical ventilation (IMV)) and death, and to assess the magnitude of the association and the potential dose-response relationships.

\section{METHODS}

\section{Protocol writing and registration}

The protocol for this review was registered with the International Prospective Register of Systematic Reviews (identification number CRD42020198718). This systematic review was conducted based on the Preferred Reporting Items for Systematic Reviews and Meta-Analyses statement. $^{9}$

\section{Search strategy}

We searched PubMed, Embase, Cochrane, Web of Sciences, WHO COVID-19 database, Google Scholar, followed by a hand search of the reference lists of related articles and reviews for studies published up to 20 July 2021. The search strategy included a combination of keywords and Medical Subject Headings (MeSH) for the concepts of COVID-19 and obesity. The following search strategy was used in PubMed and then adapted to other databases: (Covid-19 OR corona virus disease 2019 OR Novel corona virus OR novel corona OR SARS Cov-2 OR severe acute respiratory syndrome corona virus-2 OR 2019 novel coronavirus disease OR COVID-19 pandemic OR SARS-CoV-2 infection OR COVID-19 virus disease OR 2019 novel coronavirus infection OR 2019-nCoV infection OR coronavirus disease 2019 OR coronavirus disease-19 OR 2019-nCoV disease OR COVID-19 virus infection OR novel corona OR Wuhan coronavirus OR 2019 novel coronavirus OR 2019-nCoV OR Wuhan seafood market pneumonia virus OR COVID 19 virus OR coronavirus disease 2019 virus OR 'SARS-CoV-2' [MeSH] OR 'COVID19 ' $[\mathrm{MeSH}]$ ) AND (Obesity OR BMI OR Overweight OR over nutrition OR fat OR adiposity OR Body Mass index OR Weight OR height OR obese OR underweight OR normal weight OR malnutrition OR nutritional status OR Neck circumference OR Waist circumference OR Waist to hip ratio OR Visceral Adipose Tissue OR 'obesity' [MeSH Terms] OR 'overweight' [MeSH Terms] OR 'nutritional status' [MeSH Terms] OR 'adipose tissue' [MeSH Terms] OR 'body mass index' [MeSH Terms] OR 'malnutrition' [MeSH Terms] OR 'waist circumference' [MeSH Terms] OR 'intra-abdominal fat' [MeSH Terms] OR 'waist-hip ratio' [MeSH Term]). Initial study eligibility was assessed by reviewing titles and abstracts and was carried out independently by two of the authors (WS/ MT). These two authors then independently carried out full-text screening, with any disagreement being resolved by consulting a third author (TA).

\section{Inclusion/exclusion criteria}

The inclusion criteria included (1) cohort studies (prospective and retrospective), case-control studies and cross-sectional studies, (2) studies with effect estimates on the association between overweight or obesity and COVID-19 outcomes, (3) written in English, (4) peer reviewed, and (5) adjusted for at least one covariate.

The exclusion criteria were (1) studies conducted in non-human subjects or in vitro, (2) infection of unspecific coronaviruses or other respiratory viruses, (3) duplicate studies or overlapping data, (4) articles without available full text or abstract only, and (5) commentaries and reviews.

\section{Data extraction}

We developed a standardised form to extract information from each study. The main components were the study characteristics (title, author, country, journal, publication date), the study design (design, sample size, participants, outcomes and exposure definition, covariates), the results (effect estimates and 95\% CI) and limitations. Corresponding authors whose articles lack the above details were contacted via email for further information. Data were extracted by two authors (WS/MT) and verified by a third author (AG). Where multiple studies used the same data source, we included the study with the most recent data.

\section{Exposure variable}

BMI and non-BMI measures were used as indicators to reflect obesity status. Non-BMI-defined obesity included neck circumference, waist circumference, waist-to-hip ratio and visceral adipose tissue. BMI-defined obesity included BMI as a continuous variable and categorised BMI. The categorisation was based on the geographic location of the included studies. For studies conducted in Asian-Pacific countries, ${ }^{10}$ normal weight was defined as BMI (18.5-22.9 kg/m²), overweight BMI (23-24.9 kg/ $\mathrm{m}^{2}$ ), obese BMI $\geq 25 \mathrm{~kg} / \mathrm{m}^{2}$ and extreme obese BMI $\geq 30$ $\mathrm{kg} / \mathrm{m}^{2}$. Similarly, for studies conducted in non-AsianPacific countries, ${ }^{11}$ normal weight was defined as BMI $\left(18.5-24.9 \mathrm{~kg} / \mathrm{m}^{2}\right)$, overweight BMI $\left(25-29.9 \mathrm{~kg} / \mathrm{m}^{2}\right)$, obese BMI $\geq 30 \mathrm{~kg} / \mathrm{m}^{2}$ and extreme obese BMI $\geq 40 \mathrm{~kg} /$ $\mathrm{m}^{2}$.

\section{Outcome variables}

Diagnosis with SARS-CoV-2 was defined by a positive reverse transcription polymerase chain reaction (RTPCR) assay from a nasopharyngeal sample. Three categories of outcomes were considered in the present analysis. The first category included studies that reported a single outcome such as hospital admission, ICU admission, intubation or IMV and death. The second category included studies that reported endpoints with two or more combinations of the following: hospital admission, ICU, IMV, death or other conditions pertaining to a severe disease. 


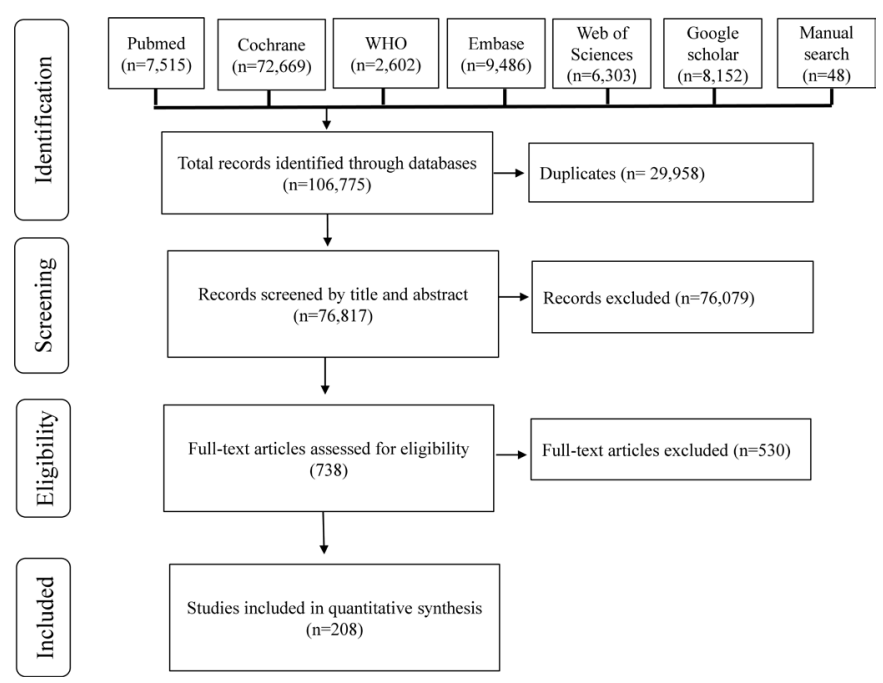

Figure 1 Flow chart of the literature selection process.

In the third category, hospital admission, ICU and IMV were pooled to provide an estimate for COVID-19-related hospitalisations.

\section{Covariates}

Based on the current knowledge of the risk factors of severe COVID-19, we identified the following covariates as most important confounders: age, sex, race/ethnicity, socioeconomic status and comorbidities (cardiovascular diseases, diabetes mellitus, chronic kidney disease, chronic pulmonary diseases).

\section{Quality assessment}

The Newcastle-Ottawa Quality Assessment Scale was used to assess the risk of bias in the included studies. One reviewer (WS) independently assessed the quality of the studies, and a second reviewer (MT) verified the evaluation. Any disagreements were resolved by consulting a third reviewer (TA).

\section{Data analysis}

We summarised the associations between obesity and COVID-19-related outcomes through a random-effects meta-analysis using the methods suggested by DerSimonian and Laird. ${ }^{12}$ RevMan V.5.4 (Cochrane Collaboration) was used to analyse the data and associated adjusted ORs and 95\% CIs were reported. Funnel plots were used to visually assess publication bias.

Studies from the general population were analysed separately from studies of restricted (by age, sex, race/ ethnicity, disease or condition) populations. The analysis was performed for obesity categories (overweight, obese, extreme obese) compared with normal weight or non-obese, and the outcomes of hospital admission, ICU, IMV, death and combinations of outcomes. We further combined obese versus normal weight and obese versus non-obese into obese and similarly for extreme obese. Then, we visually assess dose-response relationships by plotting the pooled OR versus obesity categories. An analysis by year of publication and geographic location

\begin{tabular}{|c|c|c|}
\hline Characteristics & Studies (n) & $\%$ \\
\hline \multicolumn{3}{|l|}{ Country } \\
\hline USA & 77 & 37.02 \\
\hline France & 19 & 9.13 \\
\hline China & 17 & 8.17 \\
\hline Italy & 14 & 6.73 \\
\hline England & 14 & 6.73 \\
\hline Mexico & 11 & 5.29 \\
\hline Multi-county & 8 & 3.85 \\
\hline Brazil & 7 & 3.37 \\
\hline Spain & 6 & 2.88 \\
\hline Netherlands & 4 & 1.92 \\
\hline Korea & 3 & 1.44 \\
\hline $\begin{array}{l}\text { Germany, Ireland, Kuwait, } \\
\text { Saudi Arabia, Turkey }\end{array}$ & 2 & 0.96 \\
\hline $\begin{array}{l}\text { Argentina, Australia, Chile, } \\
\text { Colombia, Democratic } \\
\text { Republic of Congo, Egypt, } \\
\text { Iran, Israel, Japan, Libya, } \\
\text { Morocco, Peru, Qatar, } \\
\text { Scotland, Singapore, } \\
\text { Switzerland, Thailand }\end{array}$ & 1 & 0.48 \\
\hline \multicolumn{3}{|l|}{ Study design } \\
\hline Prospective cohort & 35 & 16.83 \\
\hline Retrospective cohort & 158 & 75.96 \\
\hline Case-control & 5 & 2.4 \\
\hline Cross-sectional & 10 & 4.8 \\
\hline \multicolumn{3}{|l|}{ Study participants } \\
\hline General population & 168 & 80.77 \\
\hline Restricted population* & 40 & 19.23 \\
\hline \multicolumn{3}{|l|}{ Publication } \\
\hline Studies published in 2020 & 129 & 62.02 \\
\hline Studies published in 2021 & 79 & 37.98 \\
\hline
\end{tabular}

${ }^{*}$ Restricted by age, sex, race/ethnicity, disease or condition.

was also performed. In a sensitivity analysis, we further restricted the data to cohort studies (prospective and retrospective) and prospective cohort studies.

\section{RESULTS}

\section{Description of included studies}

After a full-text assessment, 208 studies were included in this meta-analysis (figure 1). The characteristics of the included studies are presented in table 1 and online supplemental table 1. A total of 3550977 participants from over 32 countries were included in this study. Seventy-seven studies were conducted in the USA, 19 in France, 17 in China, 14 in Italy, 14 in England, 11 in Mexico, 7 in Brazil and 6 in Spain. Ten studies were crosssectional, 5 case-control and 193 cohort. Forty studies 


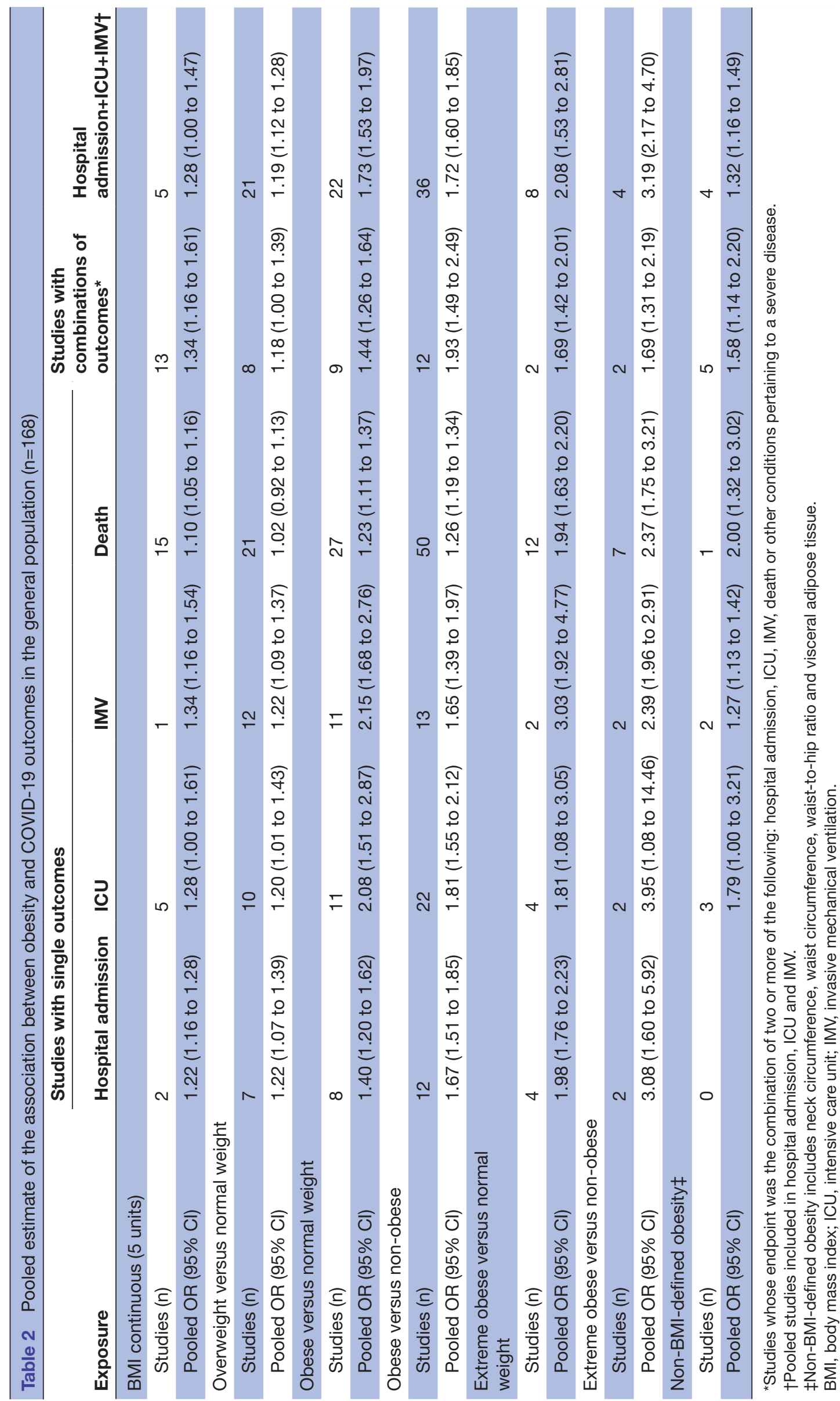

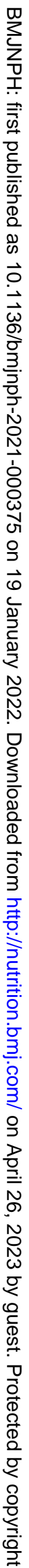


Table 3 Pooled estimate of the association between obesity and COVID-19 outcomes in restricted (by age, sex, race/ ethnicity, disease or condition) populations $(n=40)$

\begin{tabular}{|c|c|c|c|}
\hline Exposure & Hospital admission+ICU+IMV* & Death & $\begin{array}{l}\text { combinations of } \\
\text { outcomest }\end{array}$ \\
\hline \multicolumn{4}{|l|}{ BMI continuous (5 units) } \\
\hline Studies (n) & 4 & 6 & 2 \\
\hline Pooled OR $(95 \% \mathrm{Cl})$ & 1.16 (1.05 to 1.28$)$ & 1.16 (1.05 to 1.34$)$ & 1.69 (0.70 to 4.16$)$ \\
\hline \multicolumn{4}{|c|}{ Overweight versus normal weight } \\
\hline Studies (n) & 5 & 5 & 3 \\
\hline Pooled OR (95\% Cl) & $1.03(0.90$ to 1.17$)$ & 1.01 (0.88 to 1.16$)$ & 1.51 (0.89 to 2.58$)$ \\
\hline \multicolumn{4}{|c|}{ Obese versus normal weight } \\
\hline Studies (n) & 5 & 5 & 2 \\
\hline Pooled OR (95\% Cl) & $1.16(0.92$ to 1.45$)$ & 1.03 (0.78 to 1.37$)$ & 1.33 (0.99 to 1.80$)$ \\
\hline \multicolumn{4}{|l|}{ Obese versus non-obese } \\
\hline Studies (n) & 6 & 9 & 8 \\
\hline Pooled OR (95\% Cl) & $1.84(1.43$ to 2.37$)$ & 1.88 (1.61 to 2.19$)$ & 1.67 (1.29 to 2.15$)$ \\
\hline \multicolumn{4}{|c|}{ Extreme obese versus normal weight } \\
\hline Studies (n) & 2 & 2 & 1 \\
\hline Pooled OR (95\% Cl) & 1.33 (0.98 to 1.79$)$ & 1.40 (1.13 to 1.72$)$ & 1.22 (0.67 to 2.21$)$ \\
\hline \multicolumn{4}{|c|}{ Extreme obese versus non-obese } \\
\hline Studies (n) & 0 & 0 & 1 \\
\hline Pooled OR (95\% Cl) & & & $4.10(1.28$ to 13.11$)$ \\
\hline \multicolumn{4}{|l|}{ Non-BMI-defined obesitył } \\
\hline Studies (n) & 0 & 1 & 1 \\
\hline Pooled OR (95\% Cl) & & 10.71 (1.57 to 73.06$)$ & 1.25 (0.98 to 1.59$)$ \\
\hline
\end{tabular}

*Pooled studies included in-hospital admission, ICU and IMV. The estimates for hospital admission, ICU and IMV were not shown separately because of low number of studies.

†Studies whose endpoint was the combination of two or more of the following: hospital admission, ICU, IMV, death or other conditions pertaining to a severe disease.

$\ddagger$ Non-BMI-defined obesity includes neck circumference, waist circumference, waist-to-hip ratio and visceral adipose tissue.

$\mathrm{BMI}$, body mass index; ICU, intensive care unit; IMV, invasive mechanical ventilation.

were conducted in a restricted population and 168 in the general population.

\section{Association between obesity categories and COVID-19 outcomes}

Our results indicate that overweight was associated with higher odds of hospital admission (OR 1.22, 95\% CI 1.07 to 1.39 , $\mathrm{n}=7$ studies), ICU (OR $1.20,95 \%$ CI 1.01 to 1.43 , $\mathrm{n}=12$ ) and IMV (OR $1.22,95 \%$ CI 1.09 to $1.37, \mathrm{n}=12$ ), giving a significant pooled OR for COVID-19-related hospitalisations (OR 1.19, 95\% CI 1.12 to $1.28, \mathrm{n}=21$ ) (table 2 and online supplemental figure 1). However, there was no association between overweight and COVID-19 death (OR 1.02, 95\% CI 0.92 to $1.13, \mathrm{n}=21$ ). Likewise, in the restricted population analysis (table 3), overweight was not associated with COVID-19-related hospitalisations (OR 1.03, 95\% CI 0.90 to $1.17, \mathrm{n}=5$ ) and death (OR $1.01,95 \%$ CI 0.88 to $1.16, \mathrm{n}=5$ ).

Patients with obesity were at a high risk of COVID-19related hospitalisations (OR 1.73, 95\% CI 1.53 to 1.97, $\mathrm{n}=22$ ) and death (OR $1.23,95 \%$ CI 1.11 to $1.37, \mathrm{n}=27$ ) compared with normal weight (table 2 and online supplemental figure 1). Similar trends were observed comparing obese with non-obese, extreme obese with normal weight, extreme obese with non-obese and in non-BMI-defined obesity. Overall, the association between obesity categories and COVID-19 outcomes was stronger for COVID19-related hospitalisations than death. In addition, the association was stronger in the general population than restricted population.

\section{Dose-response association}

We found that every 5-unit increase in BMI was associated with $28 \%$ increased odds of COVID-19-related hospitalisations (OR 1.28, 95\% CI 1.00 to $1.47, \mathrm{n}=5$ ) and $10 \%$ increased odds of death (OR $1.10,95 \%$ CI 1.05 to $1.16, \mathrm{n}=15)$ suggesting a linear dose-response association (table 2 and online supplemental figure 1). The dose-response analysis confirmed that obesity categories were associated with COVID-19 outcomes in a linear 

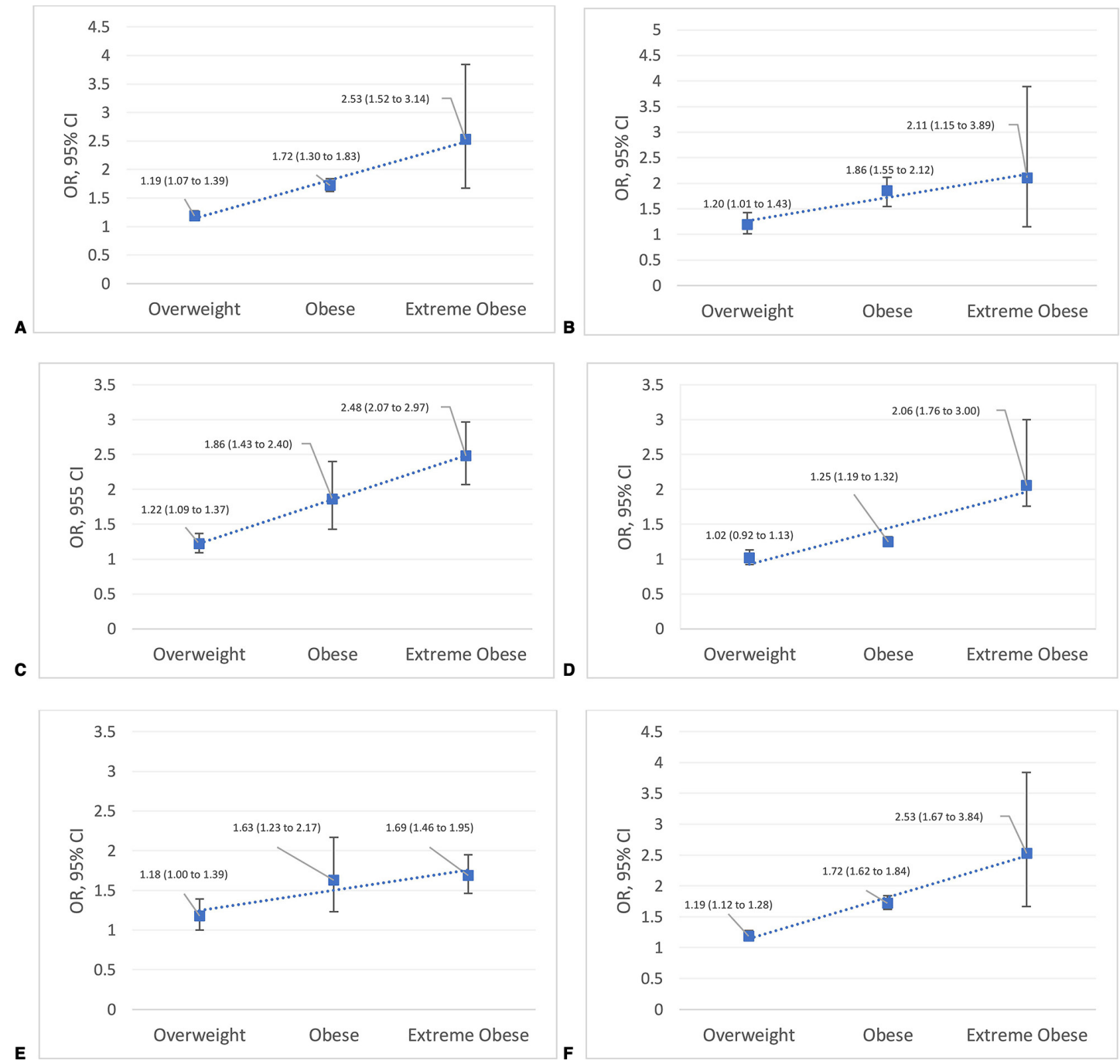

Figure 2 Dose-response association between obesity categories and COVID-19 outcomes. A) Hospital admission, B) ICU, C) IMV, D) Death, E) combinations of outcomes, F) Hospital admission + ICU + IMV. ICU, intensive care unit; IMV, invasive mechanical ventilation or intubation. Combinations of outcomes regroups studies whose endpoint was the combination of two or more of the following: hospital admission, ICU, IMV, death or other conditions pertaining to a severe disaese. Hospital admission + ICU + IMV is the pooled estimate of studies included in hospital admision, ICU and IMV.

dose-response manner (figure 2). The risk of poor COVID-19 outcomes steadily increased with increasing levels of obesity.

\section{Low strength of the association over time}

Studies with the most recent data showed a weaker association between obesity categories and COVID-19 outcomes compared with the earlier days of the pandemic. For example, comparing patients with obesity to normal weight, the adjusted OR for death was 1.31 (95\% CI 1.10 to $1.57, \mathrm{n}=17$ ) in studies published in 2020 and $1.17(95 \%$ CI 1.03 to $1.32, \mathrm{n}=10)$ in studies published in 2021 (table 4). Furthermore, the strength of the association decreased following the pattern of the first wave of COVID-19 spread. For example, the association between obesity and COVID-19 death was 1.73 (95\% CI 1.36 to 2.20, $\mathrm{n}=5$ ) in Asian-Pacific countries, 1.43 (95\% CI 1.14 to 1.79, $\mathrm{n}=24)$ in European countries and 1.13 (95\% CI 1.04 to $1.22, \mathrm{n}=31$ ) in North American countries (table 5).

\section{Sensitivity analysis}

The analysis from the general population further restricted to cohort studies (online supplemental table 2) and prospective cohort studies (online supplemental table 3) were consistent with the main analysis. In both analyses, being overweight was associated with an increased risk of COVID-19-related hospitalisations (OR $1.19,95 \%$ CI 1.12 to $1.28, \mathrm{n}=20$ and OR $1.30,95 \%$ CI 1.08 to $1.57, \mathrm{n}=1$ ), but not death (OR $1.00,95 \%$ CI 0.90 to $1.10, \mathrm{n}=20$ and OR $1.09,95 \%$ CI 0.94 to $1.26, \mathrm{n}=6$, respectively). Obesity and extreme obesity remained associated with COVID-19-related hospitalisations and death.

\section{Assessment of quality and publication bias}

All included studies had a quality score from the Newcastle-Ottawa Quality Assessment Scale $\geq 6$, as shown in online supplemental table 1 , which is acceptable for a meta-analysis. No outstanding bias was found from 
Table 4 Pooled estimate of the association between obesity and COVID-19 outcomes in the general population by publication year $(n=168)$

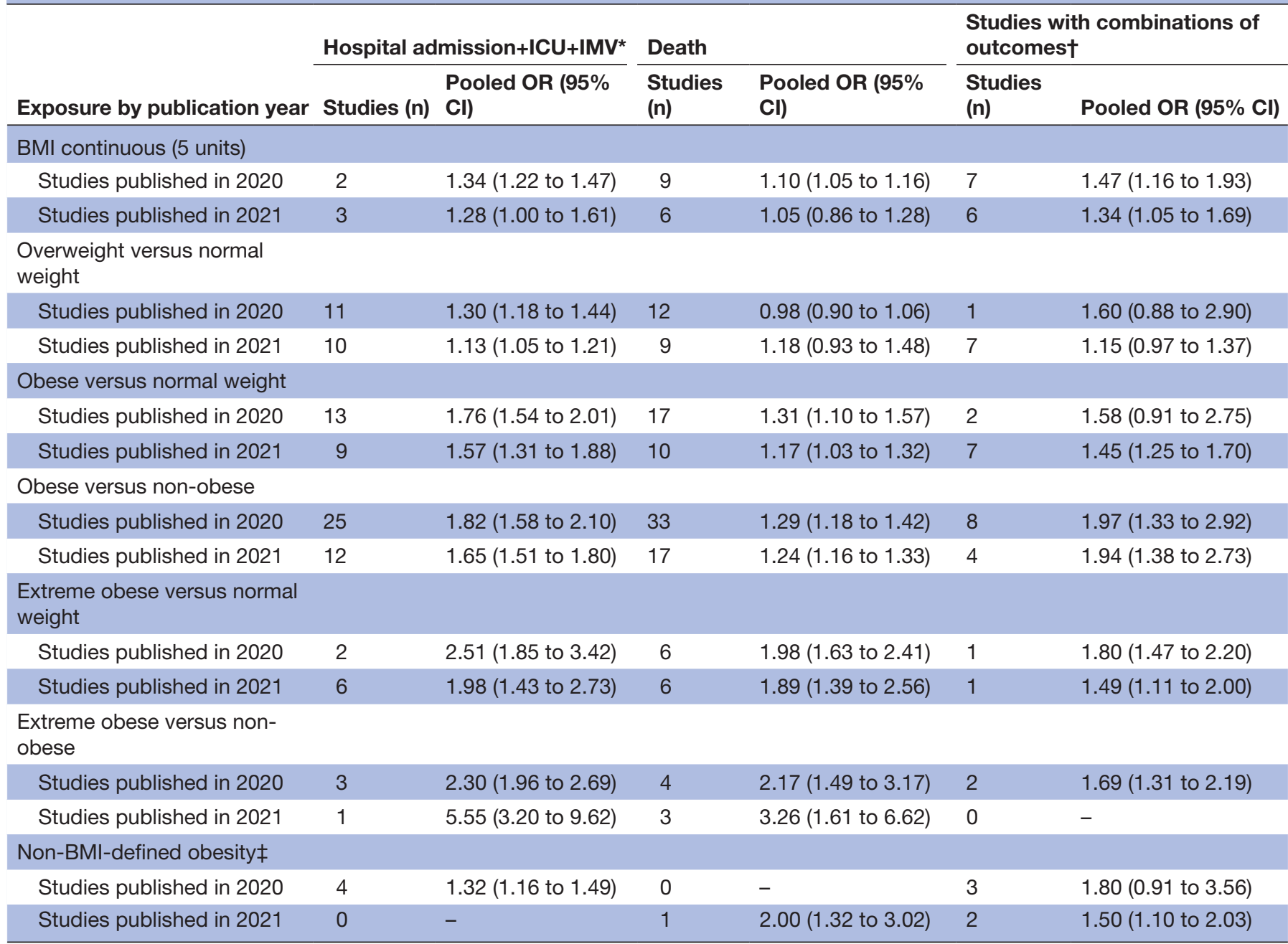

*Pooled studies included in-hospital admission, ICU and IMV. The estimates for hospital admission, ICU and IMV were not shown separately because of low number of studies.

†Studies whose endpoint was the combination of two or more of the following: hospital admission, ICU, IMV, death or other conditions pertaining to a severe disease.

$\ddagger$ Non-BMl-defined obesity includes neck circumference, waist circumference, waist-to-hip ratio and visceral adipose tissue. $\mathrm{BMI}$, body mass index; ICU, intensive care unit; IMV, invasive mechanical ventilation.

a visual inspection of the funnel plots (online supplemental figure 2).

\section{DISCUSSION}

We found that being overweight increases the risk of COVID-19-related hospitalisations but not death while obesity and extreme obesity increase the risk of both COVID-19-related hospitalisations and death. In addition, there was a linear dose-response association between obesity categories and COVID-19 outcomes. However, the strength of the association has weakened over time following the pattern of the first wave of COVID-19.

We included studies that used BMI as a continuous variable. To the best of our knowledge, this is the first metaanalysis to summarise studies that reported a continuous BMI. We found that the pooled estimate was consistent with the findings from the categorised BMI and the doseresponse analysis. This finding of dose-response relationships should inform future causation studies.

In the analysis restricted to the general population, we reached statistical significance for overweight and hospital admission, ICU and IMV, but not death. Yang et al found similar results concerning hospital admission (OR 2.09, 95\% CI 1.22 to 3.60), IMV (OR $1.40,95 \%$ CI 1.13 to 1.73 ) and death (OR $1.10,95 \%$ CI 0.84 to 1.45 ), but did not reach statistical significance for ICU admission (OR 3.10, 95\% CI 0.76 to 12.61$).{ }^{7}$ Another study by Yang $e t a l^{13}$ found a significant result for hospital admission (OR 1.30, 95\% CI 1.09 to 1.57 ) with only two studies and reported results for death (OR $1.06,95 \%$ CI 0.79 to 1.42) similar to our findings. In the restricted population, overweight was not associated with COVID-19-related 
Table 5 Pooled estimate of the association between obesity and COVID-19 outcomes in the general population by region $(n=150)$

\begin{tabular}{|c|c|c|c|c|c|c|}
\hline \multirow[b]{2}{*}{ Exposure by region } & \multicolumn{2}{|c|}{$\begin{array}{l}\text { Hospital } \\
\text { admission+ICU+IMV* }\end{array}$} & \multicolumn{2}{|l|}{ Death } & \multicolumn{2}{|c|}{$\begin{array}{l}\text { Studies with combinations } \\
\text { of outcomest }\end{array}$} \\
\hline & $\begin{array}{l}\text { Studies } \\
\text { (n) }\end{array}$ & $\begin{array}{l}\text { Pooled OR }(95 \% \\
\mathrm{Cl})\end{array}$ & $\begin{array}{l}\text { Studies } \\
\text { (n) }\end{array}$ & Pooled OR (95\% Cl) & $\begin{array}{l}\text { Studies } \\
\text { (n) }\end{array}$ & $\begin{array}{l}\text { Pooled OR }(95 \% \\
\text { Cl) }\end{array}$ \\
\hline \multicolumn{7}{|c|}{ BMI continuous (5 units) } \\
\hline Asian-Pacific & 0 & - & 1 & $4.32(1.05$ to 17.9$)$ & 5 & 2.49 (1.76 to 3.44$)$ \\
\hline Europe & 3 & 1.28 (1.00 to 1.61$)$ & 5 & 1.16 (1.00 to 1.28$)$ & 3 & $1.22(0.95$ to 1.54$)$ \\
\hline \multicolumn{7}{|l|}{ Overweight } \\
\hline Asian-Pacific & 1 & $0.84(0.46$ to 1.55$)$ & 1 & $0.53(0.14,2.00)$ & 3 & 1.16 (1.00 to 1.33$)$ \\
\hline Europe & 3 & 2.11 (1.28 to 3.46$)$ & 6 & 1.14 (0.93 to 1.39$)$ & 2 & $1.68(0.78$ to 3.65$)$ \\
\hline North America & 15 & 1.18 (1.10 to 1.25$)$ & 14 & 1.00 (0.88 to 1.14$)$ & 3 & $1.03(0.82$ to 1.29$)$ \\
\hline North America & 34 & $1.52(1.45$ to 1.59$)$ & 41 & $1.13(1.04$ to 1.22$)$ & 6 & 1.38 (1.18 to 1.62$)$ \\
\hline \multicolumn{7}{|l|}{ Extreme obese } \\
\hline Asian-Pacific & 0 & - & 1 & $4.32(1.37$ to 13.61$)$ & 0 & - \\
\hline Europe & 2 & 4.08 (2.13 to 7.82$)$ & 5 & $2.56(2.11$ to 3.11$)$ & 1 & $2.30(1.20$ to 4.40$)$ \\
\hline North America & 9 & 2.25 (1.91 to 2.65$)$ & 13 & 1.81 (1.57 to 2.08$)$ & 3 & 1.66 (1.44 to 1.93$)$ \\
\hline \multicolumn{7}{|c|}{ Non-BMI-defined obesity $\ddagger$} \\
\hline Asian-Pacific & 0 & - & 0 & - & 1 & 2.47 (1.02 to 5.98$)$ \\
\hline Europe & 4 & 1.32 (1.16 to 1.49$)$ & 0 & - & 3 & $1.63(1.00$ to 2.66$)$ \\
\hline North America & 0 & - & 1 & 2.00 (1.32 to 3.02$)$ & 1 & 1.28 (0.92 to 1.79$)$ \\
\hline
\end{tabular}

*Pooled studies included in-hospital admission, ICU and IMV. The estimates for hospital admission, ICU and IMV were not shown separately because of low number of studies.

†Studies whose endpoint was the combination of two or more of the following: hospital admission, ICU, IMV, death or other conditions pertaining to a severe disease.

$\ddagger$ Non-BMI-defined obesity includes neck circumference, waist circumference, waist-to-hip ratio and visceral adipose tissue.

BMI, body mass index; ICU, intensive care unit; IMV, invasive mechanical ventilation.

outcomes. This null finding is probably due to the heterogeneity of this group.

We found a significant association between obesity and extreme obesity with hospital admission, ICU, IMV and death. These results are consistent with the previous meta-analysis. ${ }^{78}$ However, we noticed a reduction in the strength of the association in general compared with previous studies. ${ }^{7813}$ This might be due to our inclusion criteria which included only peer-reviewed studies that adjusted for at least one confounder. The relation between obesity and COVID-19 outcomes is confounded by variables such as age, sex and comorbidities, and adjusting for these variables could reduce the effect estimate. Additionally, studies with the most recent data showed a weaker association between obesity and COVID-19 outcomes compared with the earlier days of the pandemic. As the disease progressed, there has been an improvement in the care including better healthcare facilities preparedness, knowledge, and experience on clinical management, the finding of new drugs and increased public awareness about the disease transmission and prevention.

Our finding of the association between non-BMIdefined obesity and COVID-19 outcomes adds to the literature. Systematic reviews including non-BMI obesity are rare with the highest number of included studies being $4 .{ }^{14} \mathrm{BMI}$, which is widely used to assess obesity, is often criticised as not reflecting an accurate adiposity status. Therefore, including studies that used non-BMI-defined obesity strengthens our results.

The underlying mechanism by which obesity could increase the risk of severe COVID-19 has been discussed in numerous publications. ${ }^{5615}$ First, adipose tissue could be a reservoir for viral production. This is because obesity is known to increase ACE2 expression which facilitates the cellular entry of SARS-CoV-2. Second, obesity is linked to impaired immune function which weakens the body that fails to contain viral replication. Third, obesity increases inflammation, which may have effects on the lung parenchyma and bronchi. Fourth, obesity decreases 
lung capacity and reserve and can make ventilation more difficult.

This study is the largest and most comprehensive summary of the association between obesity and COVID-19 outcomes to date. We included BMI-defined and non-BMI-defined obesity and performed a doseresponse, a geographic and publication period analysis. However, this study was not without limitations. First, the number of eligible studies under the extremely obese category and non-BMI-defined obesity was limited which has impacted the precision of the pooled estimate resulting in large CIs. Second, in the effort to reduce overlapping data, we excluded six large studies, but we could not rule out the possibility of residual participants' overlap. Third, obesity status was dichotomised in some studies which could result in extreme obese participants being included in the obese group. Fourth, heterogeneity was high in the evaluated outcomes as expected. In addition, non-BMIdefined obesity studies were based on different scales and combining them might yield residual bias. Finally, most included studies were retrospective cohort and a causal relationship between obesity and COVID-19 outcomes would require further investigations.

\section{CONCLUSION}

This systematic review and meta-analysis revealed that overweight, obesity and extreme obesity increase the risk of poor COVID-19 outcomes in a dose-response manner. However, the magnitude of the association has weakened over time following the pattern of COVID-19 spread. Nevertheless, excess adiposity remains a significant risk factor of poor COVID-19 outcomes and required more attention. These findings suggest the importance of increased vigilance towards people with excess adiposity. Prompt access to COVID-19 testing and healthcare, prioritisation for COVID-19 vaccination and other preventive measures are warranted for this vulnerable group.

Acknowledgements We thank John W Cyrus for his support in developing the search strategies and Joe Lerch who assisted in proofreading the manuscript.

Contributors WS, MT and TA conceived the study and developed the protocol. WS and MT did the literature search and selected the studies, and TA resolved the disagreement. WS and MT extracted the data, and AG verified the data. WS synthesised the data. WS drafted the initial report with input from MT, AG and TA. WS, MT, AG and TA critically revised the successive drafts of the paper. All authors approved the final version of the report and take responsibility for the integrity of the data and the accuracy of the data analysis.

Funding The authors have not declared a specific grant for this research from any funding agency in the public, commercial or not-for-profit sectors.

Competing interests None declared.

Patient consent for publication Not required.
Ethics approval This study does not involve human participants.

Provenance and peer review Not commissioned; externally peer reviewed.

Data availability statement All data relevant to the study are included in the article or uploaded as supplementary information.

Supplemental material This content has been supplied by the author(s). It has not been vetted by BMJ Publishing Group Limited (BMJ) and may not have been peer-reviewed. Any opinions or recommendations discussed are solely those of the author(s) and are not endorsed by BMJ. BMJ disclaims all liability and responsibility arising from any reliance placed on the content. Where the content includes any translated material, BMJ does not warrant the accuracy and reliability of the translations (including but not limited to local regulations, clinical guidelines, terminology, drug names and drug dosages), and is not responsible for any error and/or omissions arising from translation and adaptation or otherwise.

Open access This is an open access article distributed in accordance with the Creative Commons Attribution Non Commercial (CC BY-NC 4.0) license, which permits others to distribute, remix, adapt, build upon this work non-commercially, and license their derivative works on different terms, provided the original work is properly cited, appropriate credit is given, any changes made indicated, and the use is non-commercial. See: http://creativecommons.org/licenses/by-nc/4.0/.

ORCID iD

Wendemi Sawadogo http://orcid.org/0000-0002-6114-3641

\section{REFERENCES}

1 Cucinotta D, Vanelli M. Who Declares COVID-19 a pandemic. Acta Biomed 2020;91:157-60.

2 Dyer O. Covid-19: delta infections threaten herd immunity vaccine strategy. BMJ 2021;374:n1933.

3 Worldometer. Available: https://www.worldometers.info/coronavirus/ [Accessed 14 Sep 2021].

4 Sepandi M, Taghdir M, Alimohamadi Y, et al. Factors associated with mortality in COVID-19 patients: a systematic review and metaanalysis. Iran J Public Health 2020;49:1211-21.

5 Sattar N, Mclnnes IB, McMurray JJV. Obesity is a risk factor for severe COVID-19 infection: multiple potential mechanisms. Circulation 2020;142:4-6.

6 Dicker D, Bettini S, Farpour-Lambert N, et al. Obesity and COVID-19: the two sides of the coin. Obes Facts 2020;13:430-8.

7 Yang J, Ma Z, Lei Y. A meta-analysis of the association between obesity and COVID-19. Epidemiol Infect 2020;149:e11.

8 Huang Y, Lu Y, Huang Y-M, et al. Obesity in patients with COVID-19: a systematic review and meta-analysis. Metabolism 2020:113:154378

9 Moher D, Liberati A, Tetzlaff J, et al. Preferred reporting items for systematic reviews and meta-analyses: the PRISMA statement. PLoS Med 2009;6:e1000097.

10 Pan W-H, Yeh W-T. How to define obesity? Evidence-based multiple action points for public awareness, screening, and treatment: an extension of Asian-Pacific recommendations. Asia Pac J Clin Nutr 2008; 17:370-4.

11 Donato KA. Executive summary of the clinical guidelines on the identification, evaluation, and treatment of overweight and obesity in adults. Arch Intern Med 1998;158:1855-67.

12 DerSimonian R, Laird N. Meta-Analysis in clinical trials. Control Clin Trials 1986;7:177-88.

13 Yang J, Tian C, Chen Y, et al. Obesity aggravates COVID-19: an updated systematic review and meta-analysis. J Med Virol 2021;93:2662-74.

14 Földi M, Farkas N, Kiss S, et al. Visceral adiposity elevates the risk of critical condition in COVID-19: a systematic review and metaanalysis. Obesity 2021;29:521-8.

15 Simonnet A, Chetboun M, Poissy J, et al. High prevalence of obesity in severe acute respiratory syndrome Coronavirus-2 (SARS-CoV-2) requiring invasive mechanical ventilation. Obesity 2020;28:1195-9. 